\title{
Simulación de gasificación de biomasa enriquecida con hidrocarburos
}

\section{Simulation of gasification of hydrocarbons-enriched biomass}

\author{
Andrey Caballero-Chavarría', Maybi Patricia Rojas-Soto², \\ Melissa María Villalobos-Barquero ${ }^{3}$, Alexander Davis-Barquero ${ }^{4}$, \\ Carlos Roldan-Villalobos ${ }^{5}$, Róger Moya ${ }^{6}$, Allen Puente-Urbina ${ }^{7}$
}

Fecha de recepción: 21 de diciembre de 2018

Fecha de aceptación: 3 de abril de 2019

Caballero-Chavarría, A; Rojas-Soto M; Villalobos-Barquero, M; Davis-Barquero, A; Roldán-Villalobos, C; Moya, R; Puente-Urbina, A. Simulación de gasificación de biomasa enriquecida con hidrocarburos. Tecnología en Marcha. Vol. 32-4. Octubre-Diciembre 2019. Pág 60-68.

doi) https://doi.org/10.18845/tm.v32i4.4791

1 Centro de Investigación y de Servicios Químicos y Microbiológicos (CEQIATEC), Escuela de Química, Instituto Tecnológico de Costa Rica. Costa Rica. Correo electrónico: acaballero@tec.ac.cr.

(iD https://orcid.org/0000-0002-5584-8821

2 Centro de Investigación y de Servicios Químicos y Microbiológicos (CEQIATEC), Escuela de Química, Instituto Tecnológico de Costa Rica. Costa Rica. Correo electrónico: mayros06@gmail.com. (iD) https://orcid.org/0000-0001-8685-9315

3 Centro de Investigación y de Servicios Químicos y Microbiológicos (CEQIATEC), Escuela de Química, Instituto Tecnológico de Costa Rica. Costa Rica. Correo electrónico: melivillabar1593@gmail.com.

4 Escuela Ingeniería Química, Universidad de Costa Rica. Costa Rica. Correo electrónico: alexdavisb@gmail.com.

5 Escuela de Química, Instituto Tecnológico de Costa Rica. Costa Rica. Correo electrónico: croldan@tec.ac.cr.

6 Escuela de Ingeniería Forestal, Instituto Tecnológico de Costa Rica. Costa Rica. Correo electrónico: rmoya@tec.ac.cr. (iD) https://orcid.org/0000-0002-6201-8383

7 Centro de Investigación y de Servicios Químicos y Microbiológicos (CEQIATEC), Escuela de Química, Instituto Tecnológico de Costa Rica. Costa Rica. Correo electrónico: apuente@tec.ac.cr. 


\title{
Palabras clave
}

Energía alternativa; gasificación; biomasa; residuos; simulación de procesos.

\section{Resumen}

La gasificación es un proceso termoquímico en el que materias primas orgánicas se convierten en gases que contienen $\mathrm{H}_{2}$ y $\mathrm{CO}$ (gas de síntesis), los cuales pueden utilizarse para obtener productos como electricidad, combustibles y otras sustancias químicas. El proceso se encuentra influenciado por características propias del sistema donde se desarrolla la conversión térmica, las condiciones de operación y las materias primas empleadas. Se simuló un proceso de gasificación de biomasas enriquecidas con hidrocarburos en el que los gases generados son aprovechados para generación eléctrica, con tal de evaluar el uso de dichas materias primas. Se empleó un modelo de gasificación no estequiométrico, la ecuación de estado de Peng-Robinson, así como reacciones de gasificación conocidas. Se consideró un gasificador de corriente descendente trabajando con $22 \mathrm{~kg} / \mathrm{h}$ de alimentación de mezcla 3:1 biomasa:hidrocarburos, $20 \mathrm{~kg} / \mathrm{h}$ de aire (agente gasificante), 1 atm de presión absoluta y temperatura de gasificación de $900-1000^{\circ} \mathrm{C}$. Los gases generados se componen de $27,44 \% \mathrm{H}_{2}$ 39,79\% CO; 5,73\% $\mathrm{CH}_{4} ; 0,1 \% \mathrm{CO}_{2}$ y 27,01\% $\mathrm{N}_{2}$. La potencia neta del gasificador es $27,82 \mathrm{~kW}$ y el consumo de la mezcla $3: 1$ biomasa:hidrocarburos $0,79 \mathrm{~kg} / \mathrm{kWh}$. La adición de hidrocarburos a biomasa mejora las características del gas de síntesis obtenido y disminuye los requerimientos de materia prima para el funcionamiento del gasificador. La mezcla 3:1 biomasa:hidrocarburos es adecuada para el desempeño del proceso, permitiendo aprovechar residuos que de otra manera tendrían que ser dispuestos y tratados, generando beneficios energéticos, ambientales y económicos.

\section{Keywords}

Alternative energy; gasification; biomass; waste; process simulation.

\begin{abstract}
Gasification is a thermochemical process in which organic raw materials are converted into gases containing $\mathrm{H}_{2}$ and $\mathrm{CO}$ (syngas), which can be used to obtain products such as electricity, fuels and other chemicals. It is influenced by characteristics of the system where the thermal conversion takes place, the operating conditions and the raw materials used. It was simulated a process of gasification of hydrocarbons-enriched biomass in which the gases generated are used for electricity generation, to evaluate the use of such raw materials. A non-stoichiometric gasification model, the Peng-Robinson equation of state as well as known gasification reactions were used. A downdraft gasifier was considered working with $22 \mathrm{~kg} / \mathrm{h}$ of mix feed 3:1 biomass: hydrocarbons, $20 \mathrm{~kg} / \mathrm{h}$ of air (gasifying agent), $1 \mathrm{~atm}$ of absolute pressure and gasification temperature of $900-1000{ }^{\circ} \mathrm{C}$. The gases generated are composed of $27.44 \% \mathrm{H}_{2}, 39.79 \% \mathrm{CO}$, $5.73 \% \mathrm{CH}_{4}, 0.1 \% \mathrm{CO}_{2}$ and $27.01 \% \mathrm{~N}_{2}$. The net power of the gasifier is $27.82 \mathrm{~kW}$ and the consumption of the mixture 3:1 biomass:hydrocarbons $0.79 \mathrm{~kg} / \mathrm{kWh}$. The addition of hydrocarbons to biomass improves the characteristics of the syngas obtained and reduces the raw material requirements for the operation of the gasifier. The mixture 3:1 biomass:hydrocarbons is adequate for the performance of the process, allowing to take advantage of wastes that otherwise would have to be disposed of and treated, generating energy, environmental and economic benefits.
\end{abstract}




\section{Introducción}

Las sociedades modernas presentan retos importantes relacionados con una demanda energética creciente y un aumento en las emisiones de gases de efecto invernadero debido al uso de combustibles fósiles [1]. Unido a esto, diversas actividades humanas generan cantidades importantes de residuos sólidos que, de no ser gestionados adecuadamente, provocan contaminación ambiental (en aire, agua y suelo), así como proliferación de vectores que producen enfermedades [2]. Tal situación ha impulsado el desarrollo de tecnologías que permitan el aprovechamiento energético de residuos, dado a que buscan utilizar eficientemente los recursos disponibles y además disminuir el impacto en el ambiente [3, 4].

Una de las maneras de aprovechamiento de residuos sólidos para fines energéticos es por medio de procesamientos térmicos, que incluyen procesos tales como combustión, gasificación, pirólisis y carbonización hidrotérmica, entre otros [5, 6]. Dentro de estos, la gasificación es un tipo de proceso termoquímico que permite obtener gases, los cuales luego de transformaciones específicas son capaces de generar productos tales como electricidad, combustibles y otras sustancias químicas [7]. Dicho proceso se encuentra influenciado por características propias del sistema donde se desarrolla la conversión térmica, las condiciones de trabajo y las materias primas empleadas [8]. Se requiere un sustrato carbonoso, en este caso los residuos, el cual debe calentarse a temperaturas típicamente mayores a $800{ }^{\circ} \mathrm{C}$ en presencia de un agente gasificante (aire, oxígeno, $\mathrm{CO}_{2}$, entre otros), generando mezclas con diferentes composiciones que incluyen $\mathrm{CO}$ y $\mathrm{H}_{2}$ (gas de síntesis o syngas), $\mathrm{CH}_{4}, \mathrm{CO}_{2}, \mathrm{~N}_{2}$, entre otros [7, 8].

Dentro de los materiales que se pueden emplear como material de alimentación a un sistema de gasificación se encuentran biomasas lignocelulósicas residuales y residuos de hidrocarburos. Dichas materias primas deben presentar características de humedad, tamaño y flujo que permitan una operación adecuada del reactor empleado y generen productos con composiciones y poderes caloríficos adecuados [9]. Otras características de los materiales de partida incluidos en un análisis proximal, así como la composición elemental y las condiciones de operación del sistema (presión, temperatura, flujos del material de entrada y agente gasificante, entre otras) definen en gran medida los productos obtenidos [10-12].

Una de las maneras de conocer la influencia de las diferentes variables en la gasificación es mediante su simulación. Diferentes tipos de modelos pueden ser empleados, cuya aplicabilidad dependerá en gran medida de la información disponible. Dentro de estos, destaca un modelo no estequiométrico que busca la minimización de la energía libre de Gibbs (estado de equilibrio), con lo cual es posible obtener la composición de los gases de salida [10, 11, 13]. Tal información puede ser integrada con el resto de operaciones unitarias, permitiendo una descripción del proceso completo y sus limitaciones, lo cual resulta valioso para diseño, mejoramiento y optimización de procesos de gasificación [14].

En este estudio, se presenta la simulación de un proceso de gasificación en el cual los gases generados son aprovechados para generación eléctrica. El objetivo del trabajo es evaluar el uso de biomasas enriquecidas con hidrocarburos mediante el desempeño del proceso.

\section{Materiales y Métodos}

\section{Materia prima y sistema de gasificación}

La materia prima seleccionada es una mezcla de biomasa con hidrocarburos (3:1 biomasa:hidrocarburos), cuya composición se calculó a partir de sus constituyentes individuales. Se tomó como referencia el sistema de gasificación comercial Power Pallet PP20 de All Power Labs (gasificador de corriente descendente) [15]. 
Simulación del proceso de gasificación

Las simulaciones relacionadas con el proceso de gasificación seleccionado se basan en los reportes de Caballero-Chavarría [10] y Rojas-Soto [11]. Se utilizó el programa computacional Aspen Hysys V8 (Aspen Tech), considerando balances de masa y energía, así como equilibrio químico.

El proceso simulado se basa en la gasificación de la biomasa enriquecida con hidrocarburos en presencia de aire como agente gasificante (aire normalizado, libre de humedad) seguida del acondicionamiento de los gases producidos y su combustión para generación eléctrica. Se empleó un modelo de gasificación no estequiométrico y la ecuación de estado de PengRobinson, así como las reacciones de gasificación y combustión reportadas por Basu [8]. Se usaron las siguientes condiciones de operación: $22 \mathrm{~kg} / \mathrm{h}$ de alimentación, $20 \mathrm{~kg} / \mathrm{h}$ de aire (agente gasificante), 1 atm de presión absoluta y temperatura de gasificación de 900-1000 ${ }^{\circ} \mathrm{C}$.

\section{Resultados y discusión}

Con tal de establecer los lineamientos legales para la gestión integral de residuos y el uso eficiente de los recursos, Costa Rica aprobó en 2010 la Ley para la Gestión Integral de Residuos (Ley $N^{\circ}$ 8839) [16]. Esta contempla el aprovechamiento energético como una alternativa cuando evitar, reducir, reutilizar o recuperar materiales no es factible, por encima además de tratar y disponer dichos residuos [16]. Dentro de los residuos aprovechables energéticamente destacan las biomasas residuales y los residuos de hidrocarburos [10-12]. Cantidades importantes de biomasas residuales son producidas en Costa Rica debido a actividades agrícolas y agroindustriales, donde ca. 40,3\% no son aprovechadas [4]. Por su parte, residuos de hidrocarburos son residuos peligrosos generados en organizaciones con actividades relacionadas con la manipulación de combustibles, los cuales cuentan con altos contenidos energéticos [12]. Esto hace que resulte atractivo el aprovechamiento energético de biomasas residuales enriquecidas con hidrocarburos.

La materia prima considerada para este estudio es biomasa enriquecida con hidrocarburos. En este tipo de mezclas, el potencial energético aumenta con respecto a las biomasas individuales. Caballero-Chavarría [10] reporta un incremento de $42 \%$ en el poder calorífico de mezclas de pasto con residuos de hidrocarburos. Por otro lado, la composición del material de partida resulta importante ya que afecta la composición de los gases producidos en procesos de gasificación. Dentro de las reacciones involucradas, el principal componente que se emplea como reactivo es el carbono. Al incorporar hidrocarburos a la biomasa, se incrementa la cantidad de carbono y compuesto volátiles, con lo que se favorece la formación de productos deseados $\left(\mathrm{CO} \mathrm{CH}_{4}\right.$, $\mathrm{H}_{2}$ ) [8]. El cuadro 1 presenta la composición de la materia prima de trabajo calculada a partir de sus constituyentes individuales. Esta se empleó para establecer la línea de alimentación en la simulación y así obtener un flujo adecuado de gas de síntesis capaz de generar una cantidad suficiente de energía [10].

Las simulaciones se basan en el uso de un gasificador comercial de corriente descendente [15]. Este cuenta con alimentación superior para el combustible (biomasa enriquecida con hidrocarburos), alimentación lateral para el agente gasificante (aire) y salida de los productos en su parte inferior. Diferentes procesos son llevados en diferentes alturas del reactor, incluyendo secado y pirólisis por encima de la alimentación de aire, oxidación alrededor de la entrada de aire y reducción por debajo de esta [8]. El flujo de alimentación de biomasa con hidrocarburos (22 kg/h), la presión de trabajo (presión absoluta de $1 \mathrm{~atm}$ ) y la temperatura de gasificación $\left(900-1000{ }^{\circ} \mathrm{C}\right)$ se seleccionaron de acuerdo con las especificaciones del fabricante. La cantidad de agente gasificante se calculó para tener un proceso de gasificación adecuado. Esta se encuentra definida por la razón de equivalencia definida como: 
Cuadro 1. Composición calculada para una mezcla de biomasa con hidrocarburos (3:1 biomasa:hidrocarburos) [17-19]

\begin{tabular}{|c|c|}
\hline Componente & Porcentaje $(\% \mathrm{~m} / \mathrm{m})$ \\
\hline C & 56,25 \\
\hline H & 6,86 \\
\hline O & 36,0 \\
\hline S & 0,2 \\
\hline N & 0,3 \\
\hline Cenizas & 1,3 \\
\hline Agua & 10 \\
\hline
\end{tabular}

$$
R E=\frac{(A / C) r}{(A / C) e}
$$

donde $R E$ es la razón de equivalencia, $(A / C)$ r la relación real aire-combustible y $(A / C)$ e la relación estequiometria aire-combustible (para producir combustión completa). RE debe estar entre 0,2 y 0,4 para lograr un proceso de gasificación eficiente [10]. Basu [8] recomienda un valor de $R E$ de 0,25 para gasificaciones en reactores de corriente descendente, con el cual favorece la generación de $\mathrm{H}_{2}$ y $\mathrm{CO}$ (gas de síntesis), y evita la formación de carbonilla, $\mathrm{CO}_{2}$ y $\mathrm{H}_{2} \mathrm{O}$. $(A / C)$ e se puede estimar como:

$$
(A / C) e=8,89 *(\mathrm{C}+0,375 * S)+26,5 * \mathrm{H}-3,3 * 0
$$

donde $\mathrm{C}, \mathrm{S}, \mathrm{H}$ y $\mathrm{O}$ se relacionan con la composición elemental de carbono, azufre, hidrógeno y oxígeno en la materia prima de alimentación. Considerando las ecuaciones (1)-(2), la composición elemental (cuadro 1) de la mezcla de biomasa con hidrocarburos y su flujo másico de alimentación (22 kg/h), el flujo de aire calculado y empleado en la simulación fue $20 \mathrm{~kg} / \mathrm{h}$.

El gasificador considerado (de corriente descendente) permite un flujo continuo de producción de gas de síntesis, es capaz de procesar materias primas con altos contenidos de humedad, generalmente tiene diseño simple y es de bajo costo. Por otro lado, la calidad energética del gas que se obtiene con este tipo de reactores es baja, su eficiencia se ve limitada al trabajar a presión atmosférica y genera grandes cantidades de cenizas [8, 9, 20]. Sin embargo, temperaturas de $900-1000{ }^{\circ} \mathrm{C}$ favorecen la conversión sin fundir cenizas (evitando problemas operativos) y se generan gases con bajos contenidos de alquitranes que pueden ser utilizados en motores de combustión interna y turbinas de gas [10]. Además, el uso de aire como agente gasificante y presión atmosférica permiten mantener bajos costos de operación, lo cual es recomendable para sistemas de baja potencia como el gasificador propuesto [21].

El proceso simulado y los elementos usados se describen en la figura 1 y el cuadro 2. La utilización de un modelo de gasificación termodinámico no estequiométrico resulta ventajoso ya que puede llevarse a cabo su simulación conociendo la composición elemental de la alimentación y las condiciones de operación. A pesar de considerarse como un sistema en equilibrio (que genera el máximo rendimiento) en el que no se toman en cuenta aspectos como la fórmula química del material de alimentación, los mecanismos de reacción o las rapideces de reacción, se ha demostrado que este tipo de modelos permiten predecir de manera razonable 
el rendimiento y la composición de los gases producidos, dando la posibilidad de estimar otras propiedades de interés como su poder calorífico [22-24]. Adicionalmente, usando la ecuación de estado de Peng-Robinson, se determinaron otras características físicas del proceso tales como presión y temperatura en diferentes etapas.

Los principales datos obtenidos de la simulación se muestran en el cuadro 3. Couto y colaboradores [21] reportan gas de síntesis obtenido experimentalmente alimentando biomasa en un gasificador de corriente descendente con 15-21\% de $\mathrm{H}_{2}, 10-22 \%$ de $\mathrm{CO}, 1-5 \%$ de $\mathrm{CH}_{4}$, $11-13 \%$ de $\mathrm{CO}_{2}$ y el resto $\mathrm{N}_{2}$ y pequeñas cantidades de otros hidrocarburos. Comparativamente, la simulación muestra mayores porcentajes de $\mathrm{CO}, \mathrm{H}_{2}$ y $\mathrm{CH}_{4}$, así como menor porcentaje de $\mathrm{CO}_{2}$. Sin embargo, debe considerarse que las condiciones experimentales son distintas (incluyendo la composición de la materia prima, presión, temperatura, la relación de equivalencia airecombustible) además que la simulación asume sistemas en equilibrio cuando en realidad podrían presentarse procesos incompletos si los tiempos de residencia son cortos.

Las cantidades de materias primas necesarias por unidad de energía, calculadas a partir de la potencia neta del gasificador obtenida de la simulación y las características de la alimentación (flujo y composición), se presentan en el cuadro 3. Los consumos de la mezcla biomasa:hidrocarburos y biomasa son inferiores al consumo de biomasa reportado por el fabricante del gasificador considerado (1,2 kg/kWh) [15]. Esto puede atribuirse tanto al efecto del enriquecimiento de la biomasa de partida al adicionar hidrocarburos, como al hecho de considerar un proceso idealizado durante la simulación [10, 11].

A pesar de las diferencias que puedan presentarse entre simulaciones y resultados experimentales, procesos de simulación como el presentado en este estudio permiten obtener información valiosa sobre el desempeño de procesos de gasificación llevados a cabo en diferentes condiciones. Esto hace que las simulaciones sean herramientas importantes para diseño, predicción y mejoramiento de dichos procesos, así como un excelente complemento para información experimental e insumo para estimaciones económicas para implementación de proyectos [10, 11].

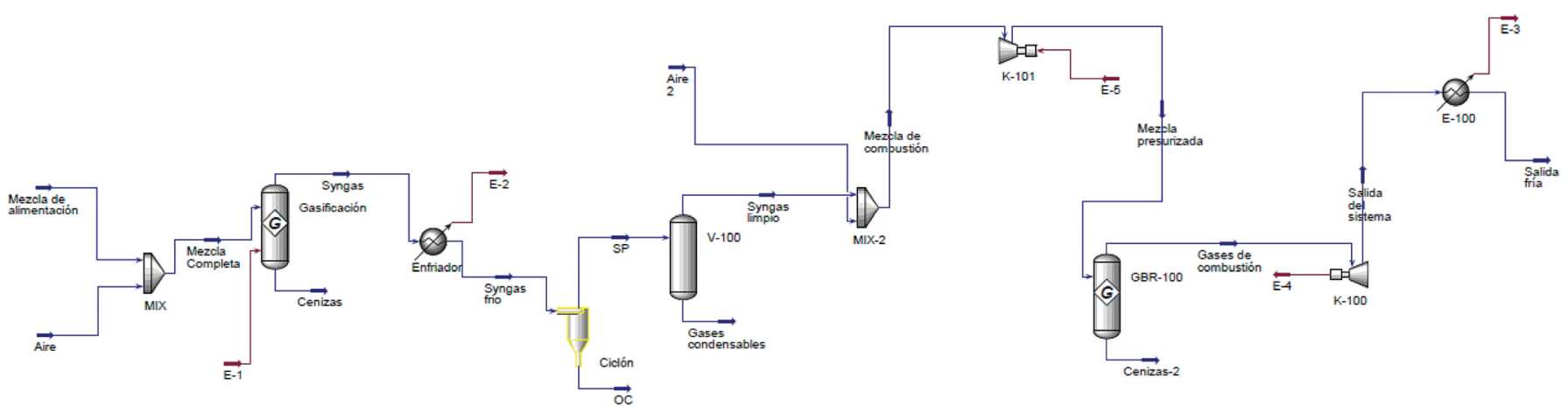

Figura 1. Esquema de simulación del proceso de gasificación empleando como materia prima la mezcla de hidrocarburos con biomasa. Basado en esquemas presentados por Caballero-Chavarría [10] y Rojas-Soto [11]. 
Cuadro 2. Descripción de elementos usados en simulación con Aspen Hysys [10, 11]

\begin{tabular}{|c|c|c|c|}
\hline Elementos & $\begin{array}{l}\text { Ejemplo de } \\
\text { elemento en } \\
\text { esquema }\end{array}$ & Funciones generales & Funciones específicas \\
\hline $\begin{array}{c}\text { Mezcladores (MIX } \\
\text { y MIX-2) }\end{array}$ & $M \mid X$ & $\begin{array}{l}\text { Mezclar dos líneas de } \\
\text { entrada. }\end{array}$ & $\begin{array}{l}\text { MIX: mezclar la alimentación de mate- } \\
\text { ria prima (biomasa enriquecida con } \\
\text { hidrocarburos) y el agente gasificante } \\
\text { (aire). } \\
\text { MIX-2: mezclar gas de síntesis (luego } \\
\text { de proceso de limpieza) y aire, gene- } \\
\text { rando la mezcla de combustión. }\end{array}$ \\
\hline $\begin{array}{l}\text { Reactor Gibbs (G } \\
\quad \text { y GBR-100) }\end{array}$ & $R-100$ & $\begin{array}{l}\text { Llevar a cabo un sistema } \\
\text { de reacciones buscan- } \\
\text { do el estado de menor } \\
\text { energía libre de Gibbs } \\
\text { y garantizando las con- } \\
\text { diciones de operación } \\
\text { necesarias. }\end{array}$ & $\begin{array}{l}\text { G: llevar a cabo el proceso de gasi- } \\
\text { ficación con todas sus etapas involu- } \\
\text { cradas. } \\
\text { GBR-100: llevar a cabo la combus- } \\
\text { tión de mezcla presurizada (mezcla } \\
\text { de gas de síntesis luego de proceso } \\
\text { limpieza y aire sometida a proceso de } \\
\text { compresión). }\end{array}$ \\
\hline $\begin{array}{c}\text { Intercambiadores } \\
\text { (Enfriador y } \\
\text { E-100) }\end{array}$ & & $\begin{array}{l}\text { Intercambiar energía en } \\
\text { porciones específicas del } \\
\text { proceso. }\end{array}$ & $\begin{array}{l}\text { Enfriador: disminuir la temperatura de } \\
\text { los gases producidos en el gasifica- } \\
\text { dor (gas de síntesis) para evitar pro- } \\
\text { blemas de operación en los equipos } \\
\text { complementarios (ciclón y columnas } \\
\text { de limpieza). } \\
\text { E-100: disminuir la temperatura de los } \\
\text { gases de salida de combustión luego } \\
\text { de expansión. }\end{array}$ \\
\hline Ciclón & Ciclón & $\begin{array}{l}\text { Separar las partículas } \\
\text { que se presentan en los } \\
\text { gases producidos en el } \\
\text { gasificador (gas de sín- } \\
\text { tesis). }\end{array}$ & $\begin{array}{l}\text { Ciclón: limpiar gases producidos en el } \\
\text { gasificador (gas de síntesis). }\end{array}$ \\
\hline $\begin{array}{l}\text { Columna } \\
\text { separadora } \\
\text { (V-100) }\end{array}$ & $V-100$ & $\begin{array}{l}\text { Reducir la presencia de } \\
\text { gases condensables que } \\
\text { pueden interferir en la } \\
\text { combustión del gas de } \\
\text { síntesis. }\end{array}$ & $\begin{array}{l}\text { V-100: limpiar el gas de síntesis pre- } \\
\text { viamente tratado en el ciclón. }\end{array}$ \\
\hline $\begin{array}{l}\text { Compresor } \\
(\mathrm{K}-101)\end{array}$ & K-101 & $\begin{array}{l}\text { Comprimir mezcla de gas } \\
\text { de síntesis luego de pro- } \\
\text { ceso limpieza y aire. }\end{array}$ & $\begin{array}{l}\text { K-101: presurizar la línea de entrada al } \\
\text { reactor de combustión. }\end{array}$ \\
\hline Expansor (K-100) & $K-100$ & $\begin{array}{l}\text { Permite simular la fun- } \\
\text { ción de una turbina y así } \\
\text { obtener la potencia del } \\
\text { sistema. }\end{array}$ & $\begin{array}{l}\text { K-100: expandir los gases de com- } \\
\text { bustión. }\end{array}$ \\
\hline
\end{tabular}


Cuadro 3. Resultados de simulación de un proceso de gasificación*

\begin{tabular}{|c|c|}
\hline Variable evaluada & Resultado \\
\hline Flujo de salida de gas de síntesis & $41,76 \mathrm{~kg} / \mathrm{h}$ \\
\hline \multirow{5}{*}{ Composición de gas de síntesis } & $\mathrm{H}_{2}: 27,44 \%$ \\
\hline & CO: $39,79 \%$ \\
\hline & $\mathrm{CH}_{4}: 5,73 \%$ \\
\hline & $\mathrm{CO}_{2}: 0,1 \%$ \\
\hline & $\mathrm{N}_{2}: 27,01 \%$ \\
\hline Potencia producida del generador & $56,32 \mathrm{~kW}$ \\
\hline Potencia consumida por el compresor & $28,50 \mathrm{~kW}$ \\
\hline Potencia aprovechable intercambiador & $81,67 \mathrm{~kW}$ \\
\hline Potencia neta del gasificador & $27,82 \mathrm{~kW}$ \\
\hline Consumos en alimentación & $\begin{array}{c}\text { 3:1 biomasa:hidrocarburos: 0,79 kg/kWh } \\
\text { Biomasa: } 0,59 \mathrm{~kg} / \mathrm{kWh} \\
\text { Hidrocarburos: } 0,20 \mathrm{~kg} / \mathrm{kWh}\end{array}$ \\
\hline
\end{tabular}

* Condiciones: gasificador de corriente descendente, $22 \mathrm{~kg} / \mathrm{h}$ de alimentación de biomasas enriquecidas con hidrocarburos (3:1 biomasa:hidrocarburos), $20 \mathrm{~kg} / \mathrm{h}$ de aire (agente gasificante), $1 \mathrm{~atm}$ de presión absoluta y temperatura de gasificación de $900-1000{ }^{\circ} \mathrm{C}$

\section{Conclusiones}

- La simulación propuesta permite obtener información relevante para la evaluación del desempeño del proceso de gasificación con las condiciones de trabajo y materias primas utilizadas.

- El gas de síntesis obtenido cuenta una composición adecuada y un bajo contenido de alquitranes que permiten su uso en dispositivos para transformar su energía química en energía mecánica y posteriormente energía eléctrica.

- La adición de hidrocarburos a biomasa permite mejorar las características del gas de síntesis obtenido y con esto disminuir los requerimientos de materia prima para el funcionamiento del gasificador.

- La mezcla de hidrocarburos y biomasa es adecuada para el desempeño del sistema de gasificación, lo cual permite aprovechar energéticamente residuos que de otra manera tendrían que ser dispuestos y tratados, generando beneficios energéticos, ambientales y económicos.

- El uso de simulaciones permite obtener información sobre el desempeño de procesos de gasificación llevados a cabo en diferentes condiciones, que puede servir de guía para el diseño, predicción y mejoramiento de dichos procesos.

\section{Agradecimientos}

A. Caballero-Chavarría, M. M. Villalobos-Barquero y A. Puente-Urbina agradecen a Paola Orozco Ballestero, Rodrigo González Rojas y Daniel Carmona Arias de RECOPE S.A., Jaime Quesada Kimzey del Instituto Tecnológico de Costa Rica y Emanuel Montero Gómez del CICAFE por valiosas discusiones. 


\section{Referencias}

[1] World Energy Outlook 2018. International Energy Agency, 2018.

[2] M. D. L. Pérez, Manual. Identificación de Residuos Industriales (UF0287). Certificados de Profesionalidad. Gestión de Residuos Urbanos e Industriales (SEAG0108). Editorial CEP, S.L., 2017.

[3] J. G. Speight, The Chemistry and Technology of Petroleum. Boca Raton: CRC press, 2014.

[4] F. Ramírez, E. Carazo, C. Roldán, and G. Villegas, "Encuesta de oferta y consumo energético nacional a partir de la biomasa en Costa Rica," Dirección Sectorial de Energía, San José, 2007.

[5] E. C. Rada, Thermochemical Waste Treatment: Combustion, Gasification, and Other Methodologies. CRC Press, 2017.

[6] M. Verma, S. Godbout, S. K. Brar, O. Solomatnikova, S. P. Lemay, and J. P. Larouche, "Biofuels production from biomass by thermochemical conversion technologies," International Journal of Chemical Engineering, vol. 2012, no. ID 542426, pp. 1-18, 2012.

[7] P. McKendry, "Energy production from biomass (part 3): gasification technologies," Bioresource Technology, vol. 83, no. 1, pp. 55-63, 2002.

[8] P. Basu, Biomass Gasification and Pyrolysis: Practical Design and Theory. Academic press, 2010.

[9] A. Heraz Segura, "Estudio de gasificación mediante un prototipo experimental para el tratamiento termoquímico de residuos orgánicos," Universidad Iberoamericana, 2012.

[10] A. Caballero-Chavarría, "Propuesta de un sistema de gasificación de residuos de hidrocarburos para aprovechamiento energético en el Plantel El Alto de RECOPE SA," Escuela de Química, Instituto Tecnológico de Costa Rica, 2017.

[11] M. P. Rojas-Soto, "Estudio de pre-factibilidad de la transformación de residuos orgánicos municipales en energía," Escuela de Química, Instituto Tecnológico de Costa Rica, 2017.

[12] M. M. Villalobos-Barquero, "Análisis de potencial de aprovechamiento energético de residuos de hidrocarburos por medio de gasificación en el Plantel El Alto de RECOPE SA," Escuela de Química, Escuela de Química Instituto Tecnológico de Costa Rica, 2017.

[13] M. Puig-Arnavat, J. C. Bruno, and A. Coronas, "Review and analysis of biomass gasification models," Renewable and Sustainable Energy Reviews, vol. 14, no. 9, pp. 2841-2851, 2010.

[14] B. Buragohain, P. Mahanta, and V. S. Moholkar, "Thermodynamic optimization of biomass gasification for decentralized power generation and Fischer-Tropsch synthesis," Energy, vol. 35, no. 6, pp. 2557-2579, 2010.

[15] ALL Power Labs. (2015). Power Pallet - PP20. Available: http://www.allpowerlabs.com/wp-content/ uploads/2015/10/PP20GeneratorOneSheet10_25_15Small.pdf

[16] Ley para la Gestión Integral de Residuos de la República de Costa Rica, Ley № 8839, 2010.

[17] M. J. Caselles Pomares, M. R. Gómez Antón, M. Molero Meneses, and J. Sardá Hoyo, Química Aplicada a la Ingeniería. Madrid: Universidad Nacional de Educación a Distancia, 2015.

[18] T. Pröll and $\mathrm{H}$. Hofbauer, " $\mathrm{H}_{2}$ rich syngas by selective $\mathrm{CO}_{2}$ removal from biomass gasification in a dual fluidized bed system — Process modelling approach," Fuel Processing Technology, vol. 89, no. 11, pp. 1207-1217, 2008.

[19] W. Doherty, A. Reynolds, and D. Kennedy, "Aspen plus simulation of biomass gasification in a steam blown dual fluidised bed," in Materials and processes for energy: communicating current research and technological developments, A. Méndez-Vilas, Ed.: Formatex Research Centre, 2013.

[20] J. U. Castellanos, F. E. Sierra Vargas, C. A. Guerrero Fajardo, and L. F. Sosa Quintero, "Modelado del proceso de gasificación de biomasa para aprovechamiento energético: una revisión al estado del arte," ITECKNE, vol. 9, no. 2, pp. 95-105, 2012.

[21] N. Couto, A. Rouboa, V. Silva, E. Monteiro, and K. Bouziane, "Influence of the biomass gasification processes on the final composition of syngas," Energy Procedia, vol. 36, pp. 596-606, 2013.

[22] A. Z. Mendiburu, J. A. Carvalho, R. Zanzi, C. R. Coronado, and J. L. Silveira, "Thermochemical equilibrium modeling of a biomass downdraft gasifier: Constrained and unconstrained non-stoichiometric models," Energy, vol. 71, pp. 624-637, 2014.

[23] B. Buragohain, P. Mahanta, and V. S. Moholkar, "Performance correlations for biomass gasifiers using semiequilibrium non-stoichiometric thermodynamic models," International Journal of Energy Research, vol. 36, pp. 590-618, 2012.

[24] T. K. Patra and P. N. Sheth, "Biomass gasification models for downdraft gasifier: A state-of-the-art review," Renewable and Sustainable Energy Reviews, vol. 50, pp. 583-593, 2015. 
Tecnología en Marcha,

Vol. 32, N. ${ }^{4}$, Octubre-Diciembre 2019 M 69 
Tecnología en Marcha,

Vol. 32, N. ${ }^{\circ}$ 4, Octubre-Diciembre 2019 